\title{
Studying the patterns of alien and native floras of some habitats in Srinagar city, Kashmir, India
}

\author{
Syed Mubashir Andrabi ${ }^{1}$, Zafar Ahmad Reshi ${ }^{1}$, Manzoor Ahmad Shah ${ }^{1 *}$ and Salman Qureshi ${ }^{2,3}$
}

\begin{abstract}
Introduction: Urban flora is an important component of human-dominated ecosystems that links urban dwellers with nature. This study aims at compiling and analysing the alien and native vascular plant species of some selected habitats in the Srinagar city, Kashmir, India.

Methods: Bi-seasonal sampling was employed and each habitat type (viz., graveyards, grasslands, orchards and wastelands) was sampled at seven different sites.

Results: The number of species recorded from graveyards was 96; from grasslands 112; 141 from orchards and 110 from wastelands. The level of invasion among habitats varied from $67.27 \%$ to $68.75 \%$, with a mean value of $67.86 \%$; out of clubbed species list, $64.84 \%$ were alien. Asteraceae, Poaceae, Brassicaceae and Fabaceae were the biggest families in all four habitat types.
\end{abstract}

Conclusions: Our research shows that across habitats, alien plant species are the predominant part of their floras.

Keywords: Alien; Flora; Herbs; Srinagar city; Level of invasion

\section{Introduction}

Important environmental services in cities like removal of dust, mitigation of microclimatic extremes and modulation of humidity are being provided by biodiversity (Bolund and Hunhammar 1999; Haase et al. 2014). The latter also delivers amenity values, such as aesthetic enjoyment and recreation (Miller 2005 and Miller 2006; Qureshi et al. 2013) and psychological benefits resulting from human biophilia (Wilson 1984). Most people live and work in cities (Miller and Hobbs 2002), and access to urban nature and natural habitats improves their quality of life (Turner et al. 2004; Qureshi 2010, Qureshi et al. 2013). Even for these people, the biotas that survive in cities are their only contact with nature (Thompson and McCarthy 2008).

But urban areas are characterised by relatively intense stress levels associated with pollution by sewage, nutrients, toxic chemicals, heat and biological pathogens (including invasive species) (Pickett et al. 2001). With ever increasing urbanisation, natural areas are becoming increasingly fragmented (Qureshi et al. 2014; Saunders et al. 1991; Hobbs and Yates 2003), creating ever more

\footnotetext{
* Correspondence: mashah75@yahoo.com

'Department of Botany, University of Kashmir, Srinagar 190 006, Jammu and Kashmir, India

Full list of author information is available at the end of the article
}

urban/wildland interfaces. These urban/wildland interfaces facilitate access and opportunities for humans to disturb natural habitats (Bolger et al. 1997), and disturbance is recognised as an important determinant of invasibility (Hobbs and Huenneke 1992; Davis 2003). Increased isolation and proliferation of edges are some features which subject the remnant natural habitats lying inter-digitated within urban landscapes (Kupfer et al. 2006) to invasibility, and the theory of island biogeography predicts fewer plant species habitation on these relatively small and isolated habitat patches (Bastin and Thomas 1999; Guirado et al. 2006).

The advantageous ability of alien plant species to grow and survive in urban areas gives them an edge over native plants which are unable to survive there (Kühn and Klotz 2006). Many alien species thrive in urban environments probably due to the 'urban heat island effect' (Ricotta et al. 2009; Wang et al. 2011). Because of the considerable potential exhibited by alien plants to disperse propagules to long distances (Pyšek and Hulme 2005), there is an understandable concern that urban areas may act as source centres from which alien plant species will spread into the wider surrounding environments (Catling and Porebski 1994; Sullivan et al. 
2005; Houlahan et al. 2006) and hence could become detrimental to regional biodiversity. A recent crosscontinental comparison revealed that invasive plants significantly reduce native plant diversity in non-native habitats but not at home (Shah et al. 2014). The invasive species can do so through many mechanisms (Levine et al., 2003), and the role of urbanisation-driven changes in ecological settings to influence such mechanisms is yet poorly understood.

Given the fact that urbanisation facilitates alien plant invasion, we carried out floristic sampling in selected habitats in Srinagar city, India and its suburbs to compile alien and native floras of these habitat types to unravel which group of plants (alien or native) are selected by regional landscape and which habit/life form is selected by the habitats?

\section{Methods}

\section{Study area, study sites and habitat types}

Srinagar city is located at an average elevation of 1,600 $\mathrm{m}$ above mean sea level and it is spread over in the heart of the oval-shaped valley of Kashmir. It is situated between $74^{\circ} 56^{\prime}$ and $75^{\circ} 79^{\prime}$ east longitude and $33^{\circ} 18^{\prime}$ and $34^{\circ} 45^{\prime}$ north latitude. Srinagar is the largest urban centre in the lap of Himalayas and its landscape is adorned by the Dal, the world-famous freshwater lake. In the east, the city is bounded by Zabarwan Mountains, hillocks of 'Takth-i-Suliman' in the east and 'Kohi-Maraan' (Hariparbat) in the centre which adds to its beauty. The famous Dachigam National Park, the last abode of the Kashmir stag 'Hanglu', lies on the eastern part of city.
All the study sites were located in Srinagar city and its suburbs. Any two study sites were at least $0.75 \mathrm{~km}$ apart. These habitats were surrounded by a typical urban matrix of residential, commercial, industrial and recreational land use. Four major terrestrial habitats selected were grasslands, graveyards, orchards and wastelands. Each habitat type (viz., graveyards, grasslands, orchards and wastelands) was sampled at seven different sites. Floristic sampling was conducted twice during the year 2010 to 2011.

\section{Data collection}

The first sampling was carried out in April and May and the second one in August 2010 and September 2011. During the present investigation, all spontaneously established vascular plant species, including garden escapes and seedlings of spontaneously regenerating planted trees and shrubs, were recorded using the stratified quadrat sampling technique (Figure 1). A quadrat size of $0.25 \mathrm{~m} \times 0.25 \mathrm{~m}$ was used for herbaceous plant species, $5 \mathrm{~m} \times 5 \mathrm{~m}$ for shrubs and $10 \mathrm{~m} \times 10 \mathrm{~m}$ for trees. A total of $210(0.25 \mathrm{~m} \times 0.25 \mathrm{~m})$ quadrats were randomly laid for herbs, $140(5 \mathrm{~m} \times 5 \mathrm{~m})$ for shrubs and $140(10 \mathrm{~m} \times 10 \mathrm{~m})$ for trees in each habitat type.

\section{Floristic element profiling: definitions and terminology}

All the recorded species were classified as either native or alien according to their status in the region i.e. Kashmir valley. The native geographical range of the plant species was obtained from every possible source, such as (Khuroo et al. 2007), Germplasm Resources Information Network (GRIN) of the US Department of Agriculture. The alien plant species were characterised at different stages of

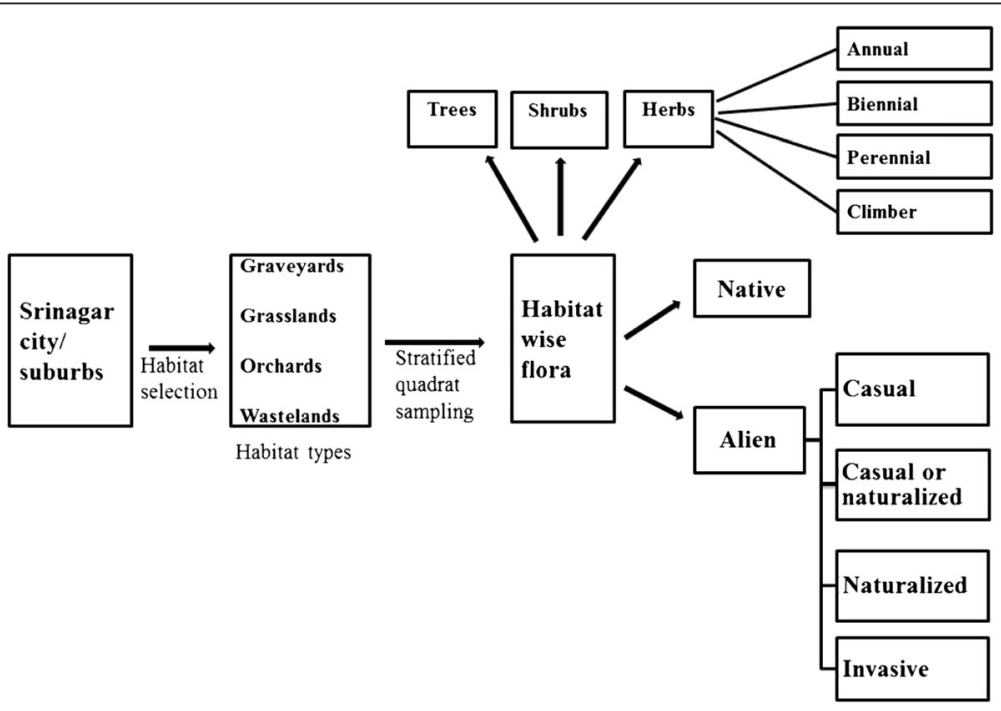

Figure 1 Schematic representation of the sequence of steps followed in the present study. Different habitat types were identified and surveyed in the Srinagar city and its suburbs. Habitat wise floras were generated using the stratified quadrant sampling approach that were further segregated into native and alien elements followed by growth form characterisation. Alien species were further categorised on the basis of stages of invasion. 
the invasion process along the introduction-naturalisationinvasion continuum (see Richardson et al. 2000) (Figure 1). In this study, we defined 'casual' alien species as those alien species that do not form self-replacing populations (Pyšek et al. 2004). Those alien species that reproduce consistently and sustain self-replacing populations over many life cycles without direct intervention by people (or in spite of human intervention) have been categorised as 'naturalised' alien species (sensu Richardson et al. 2000; Pyšek et al. 2004). 'Casual or naturalised' alien plant species were those alien plant species about which adequate field information was not available (Wu et al. 2004). We used the term 'invasive' for those alien species that cause apparent damage or pose potential threats to species, ecosystems or to the economy (IUCN (International Union for the Conservation of Nature) (1999); Wang et al. 2011). In this sense, invasives should be considered as a subset of naturalised species capable of spreading considerably and with harmful effects, a definition which could also be likened with the terms 'transformers' and 'weeds' as defined by Richardson et al. (2000) and Pyšek et al. (2004).

With regard to habit, the species were classified into trees, shrubs, sub-shrubs, lianas, climbers and herbs. The species were characterised on the basis of lifespan into various categories like annuals, biennials and perennials. The level of invasion was measured as the total numbers of alien plant species or their proportion of the total species richness (Jauni and Hyvönen 2010). Finally, the specimens bearing collection no. 200-351 SM Andrabi dated 2010 have been deposited in KASH (herbarium of the Department of Botany at the Centre for Biodiversity and Taxonomy, University of Kashmir, Srinagar).

\section{Results}

\section{Floristic diversity of specific habitat types Flora of graveyards}

The graveyard flora in urban areas comprised 96 plant species belonging to 77 genera and 32 families (Table 1). Six families namely Asteraceae (23 species), Poaceae (10 species), Brassicaceae (7 species), Fabaceae (6 species), Lamiaceae (5 species) and Apiaceae (4 species) accounted for $57.29 \%$ of plant species (Table 2). Sixteen families had one representative each in the urban graveyard flora, five families were represented by two species each and five families were represented by three species each. Out of 77 genera, only Poa and Iris were represented by three species each, 15 genera were represented by two species each and 60 genera were represented by just one species each.

\section{Growth form and life span profile of graveyard flora} Of the 96 species recorded in urban graveyards, 91 (94.79\%) were herbs (43 annual, 8 biennial, 39 perennial and 1 aquatic), and only 1 and 4 were sub-shrub and trees, respectively (Figure 2).
Out of 91 herbs recorded, 61 (67.03\%) were alien and 30 (32.97\%) were native. The only one sub-shrub species and all four tree species that were recorded were alien in the study area, and no native species from these two groups were recorded.

Proportion of alien and native species In terms of species status in the region $66(68.75 \%)$, the species were alien (Figure 3) and 30 species (31.25\%) were native. Of the 66 aliens, casuals were represented by four species, casuals or naturalised aliens were represented by just one $(1.52 \%)$ species, $24(36.36 \%)$ and $37(56.06 \%)$ plant species were grouped under naturalised and invasive categories, respectively (Table 3 ).

\section{Flora of grasslands}

The present study revealed the presence of 112 species belonging to 92 genera and 33 families (Table 1). Seven families namely Poaceae (22 species), Asteraceae (19 species), Fabaceae (10 species), Brassicaceae (6 species), Rosaceae (6 species), Scrophulariaceae (6 species), and Apiaceae (4 species) accounted for $65.18 \%$ of species (Table 2). Seventeen (51.51\%) families were each represented by one species, five families each by two species and remaining four families each were represented by three species. Out of 92 genera, only Poa and Veronica were represented by four species each and Medicago by three species. Twelve genera were each represented by two species and remaining 77 (83.69\%) genera were represented each by just one species.

Growth form and life span profile of grassland flora The analysis of the urban grassland flora revealed that 105 species belonged to herbaceous habit and accounted for about $93.75 \%$ of species. They belonged to various life forms like annuals represented by 56 species, biennials by 6 species, perennials by 40 species, aquatics by 2 species and 1 parasitic species. Two species were sub-shrubs, three were shrubs and two tree species were also recorded (Figure 2).

Out of the 105 herbs, $71(67.62 \%)$ were alien and 34 (32.38\%) were native to the Kashmir. Of the two subshrubs, one was alien and the other one was native to the region. Of the three shrubs recorded, all were alien to the Kashmir. Similarly, two tree species belonged to alien category and no native tree species was recorded from the study area.

Proportion of alien and native species Out of 112 species recorded in urban grasslands, 77 (68.75\%) were alien (Figure 3) and 35 (31.25\%) were native to the Kashmir. Out of 77 alien species, casuals included 2 species, casuals or naturalised category included 2 species, naturalised category included 33 species and $40(51.95 \%)$ species belonged to invasive category (Table 3 ). 
Table 1 Conspectus of plant species in various habitats of Srinagar city

\begin{tabular}{|c|c|c|c|c|c|c|c|c|c|}
\hline Name of plant species & Family & Group & $\begin{array}{l}\text { Species status } \\
\text { in the region }\end{array}$ & $\begin{array}{l}\text { Growth } \\
\text { form }\end{array}$ & $\begin{array}{l}\text { Invasion } \\
\text { status }\end{array}$ & Graveyard & Grassland & Orchards & Wastelands \\
\hline$\overline{\text { Achillea millefolium } \mathrm{L} .}$ & Asteraceae & Dicot & Alien & $P$ & $\ln$ & + & + & + & - \\
\hline Aegilops tauschii Cosson & Poaceae & Monocot & Alien & $A$ & $\ln$ & - & + & + & + \\
\hline Ageratum conyzoides L. & Asteraceae & Dicot & Alien & A & $\ln$ & + & - & - & - \\
\hline Ailanthus altissima Sw. & Simaroubaceae & Dicot & Alien & $\mathrm{T}$ & $\ln$ & + & - & + & + \\
\hline Althaea rosea Cav. & Malvaceae & Dicot & Alien & B & Cs & + & - & - & - \\
\hline Amaranthus caudatus L. & Amaranthaceae & Dicot & Alien & $A$ & $\ln$ & + & - & + & + \\
\hline Amaranthus spinosus L. & Amaranthaceae & Dicot & Alien & A & $\ln$ & + & + & - & + \\
\hline Anagalis arvensis $\mathrm{L}$. & Primulaceae & Dicot & Alien & A & $\ln$ & - & - & + & - \\
\hline Anchusa ovata Lehm. & Boraginaceae & Dicot & Alien & A & $\mathrm{Nt}$ & - & - & + & - \\
\hline Anthemis cotula $\mathrm{L}$ & Asteraceae & Dicot & Alien & B & $\ln$ & + & + & + & + \\
\hline Arctium lappa L. & Asteraceae & Dicot & Alien & $P$ & $\ln$ & + & + & + & + \\
\hline Arenaria serpyllifolia $\mathrm{L}$. & Caryophyllaceae & Dicot & Alien & A & In & - & + & - & - \\
\hline Artemisia absinthium L. & Asteraceae & Dicot & Alien & Ss & $\ln$ & + & + & + & + \\
\hline $\begin{array}{l}\text { Artemisia dubia Wall. } \\
\text { ex Besser }\end{array}$ & Asteraceae & Dicot & Native & $P$ & & - & - & + & - \\
\hline $\begin{array}{l}\text { Artemisia tournefortiana } \\
\text { Reichb. }\end{array}$ & Asteraceae & Dicot & Alien & A & $\mathrm{Nt}$ & + & - & + & - \\
\hline Asparagus filicinus Ham. & Liliaceae & Monocot & Native & $P$ & & - & + & + & - \\
\hline Avena sativa $\mathrm{L}$. & Poaceae & Monocot & Alien & A & Cs & - & + & + & - \\
\hline Bellis perennis $\mathrm{L}$. & Asteraceae & Dicot & Alien & $P$ & $\mathrm{Nt}$ & + & + & - & - \\
\hline Bidens cernua L. & Asteraceae & Dicot & Alien & $P$ & $\mathrm{Nt}$ & - & - & + & - \\
\hline $\begin{array}{l}\text { Bothriochloa ischaemum } \\
\text { Keng }\end{array}$ & Poaceae & Monocot & Alien & $P$ & $\ln$ & - & + & + & + \\
\hline $\begin{array}{l}\text { Brachiaria eruciformis } \\
\text { Griseb. }\end{array}$ & Poaceae & Monocot & Native & A & & - & + & - & + \\
\hline Breea arvensis Less. & Asteraceae & Dicot & Native & $P$ & & + & + & + & + \\
\hline Bromus japonicus Thunb. & Poaceae & Monocot & Alien & A & $\mathrm{Nt}$ & + & + & + & + \\
\hline Cannabis sativa $\mathrm{L}$. & Cannabiaceae & Dicot & Alien & $A$ & $\ln$ & + & + & + & + \\
\hline $\begin{array}{l}\text { Capsella bursa-pastoris } \\
\text { Medic. }\end{array}$ & Brassicaceae & Dicot & Alien & A & $\ln$ & + & + & + & + \\
\hline Cardamine hirsuta L. & Brassicaceae & Dicot & Alien & A & $\mathrm{Nt}$ & - & + & + & - \\
\hline Carex fedia Nees & Cyperaceae & Monocot & Native & $\mathrm{P}$ & & - & - & + & - \\
\hline Carex notha Kunth. & Cyperaceae & Monocot & Alien & B & $\ln$ & - & - & + & - \\
\hline Carpesium abrotanoides $\mathrm{L}$. & Asteraceae & Dicot & Alien & A & $\mathrm{Nt}$ & + & + & + & + \\
\hline Carpesium cernuum L. & Asteraceae & Dicot & Native & $P$ & & + & - & + & - \\
\hline Celtis australis L. & Ulmaceae & Dicot & Alien & $\mathrm{T}$ & $\mathrm{Nt}$ & + & - & - & + \\
\hline Cerastium vulgatum $\mathrm{L}$. & Caryophyllaceae & Dicot & Native & $P$ & & + & + & + & - \\
\hline Ceratocephalus falcatus Pers. & Ranunculaceae & Dicot & Alien & A & $\mathrm{Nt}$ & - & + & - & - \\
\hline Chenopodium album $\mathrm{L}$. & Chenopodiaceae & Dicot & Alien & A & $\ln$ & + & - & + & + \\
\hline Chenopodium botrys $\mathrm{L}$. & Chenopodiaceae & Dicot & Alien & A & $\mathrm{Nt}$ & + & + & - & + \\
\hline Chenopodium hybridum L. & Chenopodiaceae & Dicot & Alien & $A$ & $\ln$ & - & - & - & - \\
\hline Cichorium intybus L. & Asteraceae & Dicot & Alien & $P$ & $\ln$ & - & + & + & - \\
\hline Cirsium falconeri Petrak & Asteraceae & Dicot & Native & $P$ & & + & + & + & + \\
\hline $\begin{array}{l}\text { Clinopodium umbrosum } \\
\text { C. Koch }\end{array}$ & Lamiaceae & Dicot & Alien & $P$ & $\mathrm{Nt}$ & - & + & + & - \\
\hline Clinopodium vulgare $\mathrm{L}$. & Lamiaceae & Dicot & Alien & $P$ & $\mathrm{Nt}$ & + & - & + & - \\
\hline
\end{tabular}


Table 1 Conspectus of plant species in various habitats of Srinagar city (Continued)

\begin{tabular}{|c|c|c|c|c|c|c|c|c|c|}
\hline Convolvulus arvensis $\mathrm{L}$. & Convolvulaceae & Dicot & Alien & $P$ & $\ln$ & + & + & + & + \\
\hline $\begin{array}{l}\text { Conyza bonariensis } \\
\text { Cronquist }\end{array}$ & Asteraceae & Dicot & Alien & $A$ & Cs & + & - & + & + \\
\hline $\begin{array}{l}\text { Conyza canadensis } \\
\text { Cronquist }\end{array}$ & Asteraceae & Dicot & Alien & $B$ & $\ln$ & + & + & + & + \\
\hline Coronopus didymus Sm. & Brassicaceae & Dicot & Alien & A & $\mathrm{Nt}$ & + & + & + & + \\
\hline Cotula anthemoides L. & Asteraceae & Dicot & Native & A & & + & + & - & + \\
\hline Crataegus songarica K. Koch & Rosaceae & Dicot & Alien & $\mathrm{T}$ & Cs & - & + & + & - \\
\hline Crepis sancta Babc. & Asteraceae & Dicot & Alien & A & $\ln$ & - & + & + & - \\
\hline Cuscuta europaea L. & Cuscutaceae & Dicot & Native & $\mathrm{Ph}$ & & - & + & - & + \\
\hline Cynodon dactylon L. & Poaceae & Monocot & Native & $P$ & & + & + & + & + \\
\hline $\begin{array}{l}\text { Cynoglossum glochidiatum } \\
\text { Wall. ex Benth. }\end{array}$ & Boraginaceae & Dicot & Native & A & & + & + & + & + \\
\hline Cyperus iria L. & Cyperaceae & Monocot & Alien & $\mathrm{Aq}$ & $\mathrm{Nt}$ & - & + & - & - \\
\hline Cyperus rotundus L. & Cyperaceae & Monocot & Alien & $P$ & $\ln$ & + & + & + & + \\
\hline Dactylis glomerata L. & Poaceae & Monocot & Alien & $P$ & $\ln$ & - & - & + & - \\
\hline Datura stramonium L. & Solanaceae & Dicot & Alien & $A$ & $\ln$ & + & - & - & + \\
\hline Daucus carota $\mathrm{L}$. & Apiaceae & Dicot & Alien & $B$ & $\ln$ & + & + & + & - \\
\hline Delphinium roylei Munz. & Ranunculaceae & Dicot & Native & A & & - & - & - & + \\
\hline Descurainia sophia Webb. & Brassicaceae & Dicot & Alien & A & $\mathrm{Nt}$ & + & + & + & + \\
\hline Digitaria cruciata A. Camus & Poaceae & Monocot & Native & $A$ & & - & + & - & - \\
\hline $\begin{array}{l}\text { Echinochloa crus-galli P. } \\
\text { Beauv. }\end{array}$ & Poaceae & Monocot & Alien & $\mathrm{Aq}$ & $\mathrm{Nt}$ & - & + & - & + \\
\hline Echinops cornigerus DC. & Asteraceae & Dicot & Native & $P$ & & - & + & - & - \\
\hline $\begin{array}{l}\text { Equisetum debile Roxb. } \\
\text { ex Vaucher }\end{array}$ & Equisetaceae & Pteridophyte & Native & $P$ & & - & - & + & - \\
\hline $\begin{array}{l}\text { Equisetum ramosissimum } \\
\text { Desf. }\end{array}$ & Equisetaceae & Pteridophyte & Native & $P$ & & - & - & + & - \\
\hline $\begin{array}{l}\text { Erodium cicutarium L'Herit. } \\
\text { ex Ait. }\end{array}$ & Geraniaceae & Dicot & Alien & A & $\mathrm{Nt}$ & + & + & + & + \\
\hline Eryngium billardieri Del. & Asteraceae & Dicot & Native & $P$ & & + & + & + & + \\
\hline Eryngium coeruleum M-Bieb. & Asteraceae & Dicot & Native & $P$ & & + & + & + & + \\
\hline Euclidium syriacum $\mathrm{R}$. Br. & Brassicaceae & Dicot & Native & A & & - & - & + & - \\
\hline Euphorbia helioscopia L. & Euphorbiaceae & Dicot & Alien & A & $\ln$ & + & + & + & + \\
\hline Euphorbia wallichii Hook. f. & Euphorbiaceae & Dicot & Native & $P$ & & - & - & + & - \\
\hline Foeniculum vulgare Mill. & Apiaceae & Dicot & Alien & $P$ & Cs & + & - & - & + \\
\hline Fumaria indica H. N. & Fumariaceae & Dicot & Native & A & & - & + & + & + \\
\hline Galinsoga parviflora Cav. & Asteraceae & Dicot & Alien & A & $\ln$ & + & - & + & + \\
\hline Galium aparine L. & Rubiaceae & Dicot & Alien & $A$ & $\mathrm{Cn}$ & + & + & + & + \\
\hline $\begin{array}{l}\text { Garhadiolus minutissimus } \\
\text { Kitamura }\end{array}$ & Asteraceae & Dicot & Alien & A & $\mathrm{Nt}$ & - & + & - & - \\
\hline Geranium nepalense Sweet & Geraniaceae & Dicot & Native & A & & - & + & + & - \\
\hline Geranium pusillum Burm. f. & Geraniaceae & Dicot & Native & A & & + & + & + & + \\
\hline Geum roylei Bolle & Rosaceae & Dicot & Native & P & & - & - & + & - \\
\hline Herniaria hirsuta $\mathrm{L}$. & Caryophyllaceae & Dicot & Native & $P$ & & + & - & - & - \\
\hline Hesperis matronalis L. & Brassicaceae & Dicot & Alien & B & $\mathrm{Cn}$ & - & - & + & - \\
\hline Hordeum murinum $\mathrm{L}$. & Poaceae & Monocot & Native & A & & + & + & + & + \\
\hline Hypericum perforatum L. & Hypericaceae & Dicot & Alien & $P$ & $\mathrm{Nt}$ & + & + & + & - \\
\hline
\end{tabular}


Table 1 Conspectus of plant species in various habitats of Srinagar city (Continued)

\begin{tabular}{|c|c|c|c|c|c|c|c|c|c|}
\hline Imperata cylindrica P. Beauv. & Poaceae & Monocot & Alien & $P$ & $\mathrm{Nt}$ & - & + & - & - \\
\hline $\begin{array}{l}\text { Indigofera heterantha wall. } \\
\text { ex Brand. }\end{array}$ & Fabaceae & Dicot & Native & S & & - & - & + & - \\
\hline Ipomoea purpurea Roth. & Convolvulaceae & Dicot & Alien & C & $\mathrm{Nt}$ & - & - & - & + \\
\hline Iris ensata Thunb. & Iridaceae & Monocot & Alien & $P$ & $\ln$ & + & + & + & - \\
\hline Iris germanica L. & Iridaceae & Monocot & Alien & $P$ & $\mathrm{Nt}$ & + & - & - & - \\
\hline Iris kashmiriana Baker & Iridaceae & Monocot & Native & $P$ & & + & - & - & - \\
\hline Kikxia spuria Dum. & Scrophulariaceae & Dicot & Native & A & & - & + & - & - \\
\hline Lactuca serriola L. & Asteraceae & Dicot & Native & B & & + & - & + & + \\
\hline Lamium amplexicaule L. & Lamiaceae & Dicot & Native & A & & - & - & + & - \\
\hline Lathyrus aphaca L. & Fabaceae & Dicot & Alien & A & $\mathrm{Nt}$ & - & - & + & - \\
\hline Lepidium latifolium L. & Brassicaceae & Dicot & Native & $P$ & & + & - & - & - \\
\hline Lespedeza cuneata G. Don. & Fabaceae & Dicot & Native & Ss & & - & + & + & - \\
\hline $\begin{array}{l}\text { Leucanthemum vulgare } \\
\text { Lam. }\end{array}$ & Asteraceae & Dicot & Alien & $P$ & In & - & - & + & - \\
\hline Linaria dalmatica Miller & Scrophulariaceae & Dicot & Alien & $P$ & $\mathrm{Cn}$ & - & - & - & + \\
\hline Lithospermum arvense L. & Boraginaceae & Dicot & Alien & A & In & - & - & + & + \\
\hline Lolium perenne L. & Poaceae & Monocot & Alien & $P$ & $\mathrm{Nt}$ & - & + & + & + \\
\hline $\begin{array}{l}\text { Lolium persicum Boiss. and } \\
\text { Hoh. ex Boiss. }\end{array}$ & Poaceae & Monocot & Native & A & & - & - & - & + \\
\hline Lotus corniculatus L. & Fabaceae & Dicot & Alien & $P$ & $\mathrm{Nt}$ & - & + & + & + \\
\hline Malcolmia africana R. Br. & Brassicaceae & Dicot & Alien & A & $\mathrm{Nt}$ & - & - & - & + \\
\hline Malva mauritiana L. & Malvaceae & Dicot & Alien & A & Cs & - & - & + & - \\
\hline Malva neglecta Wall. & Malvaceae & Dicot & Alien & B & $\mathrm{Nt}$ & + & + & + & - \\
\hline Malva parviflora $\mathrm{L}$. & Malvaceae & Dicot & Native & A & & + & - & + & + \\
\hline Marrubium vulgare L. & Lamiaceae & Dicot & Alien & P & $\ln$ & + & + & + & + \\
\hline Mazus pumilus Van Steenis & Scrophulariaceae & Dicot & Native & A & & - & - & - & + \\
\hline Medicago lupulina L. & Fabaceae & Dicot & Alien & $P$ & $\mathrm{Nt}$ & - & + & + & - \\
\hline Medicago minima Grufb. & Fabaceae & Dicot & Native & A & & + & + & + & - \\
\hline Medicago polymorpha L. & Fabaceae & Dicot & Alien & A & $\ln$ & + & + & + & + \\
\hline Medicago sativa $\mathrm{L}$. & Fabaceae & Dicot & Alien & B & $\mathrm{Nt}$ & - & - & + & + \\
\hline Melia azedarach L. & Meliaceae & Dicot & Alien & T & Cs & + & - & - & - \\
\hline Melilotus albus Desr. & Fabaceae & Dicot & Native & A & & - & - & - & + \\
\hline Melilotus officinalis Pall. & Fabaceae & Dicot & Native & B & & - & - & - & - \\
\hline Mentha longifolia L. & Lamiaceae & Dicot & Alien & $P$ & $\ln$ & - & - & + & + \\
\hline Morus alba L. & Moraceae & Dicot & Alien & T & $\mathrm{Nt}$ & - & - & + & + \\
\hline $\begin{array}{l}\text { Muehlenbergia duthieana } \\
\text { Hack. }\end{array}$ & Poaceae & Monocot & Native & $A$ & & - & - & - & + \\
\hline $\begin{array}{l}\text { Myosotis micrantha Pall. } \\
\text { ex Lehm. }\end{array}$ & Boraginaceae & Dicot & Native & A & & - & - & + & - \\
\hline Myosotis sylvatica Hoffm. & Boraginaceae & Dicot & Native & $P$ & & - & + & - & + \\
\hline Narcissus tazetta $\mathrm{L}$. & Amaryllidaceae & Monocot & Alien & $P$ & In & + & - & - & - \\
\hline Nepeta cataria L. & Lamiaceae & Dicot & Alien & P & $\mathrm{Nt}$ & + & - & + & + \\
\hline Oenothera biennis L. & Onagraceae & Dicot & Alien & B & $\mathrm{Nt}$ & - & + & + & - \\
\hline Oenothera rosea Ait. & Onagraceae & Dicot & Alien & A & In & - & + & + & + \\
\hline Onopordum acanthium L. & Asteraceae & Dicot & Alien & $P$ & $\mathrm{Nt}$ & + & - & + & + \\
\hline Ornithogalum umbellatum L. & Hyacinthaceae & Monocot & Alien & $P$ & Cs & - & - & + & - \\
\hline
\end{tabular}


Table 1 Conspectus of plant species in various habitats of Srinagar city (Continued)

\begin{tabular}{|c|c|c|c|c|c|c|c|c|c|}
\hline Oxalis corniculata L. & Oxalidaceae & Dicot & Alien & $P$ & $\mathrm{Nt}$ & + & + & + & + \\
\hline $\begin{array}{l}\text { Papaver macrostomum } \\
\text { Boiss. \& Heut ex Boiss. }\end{array}$ & Papaveraceae & Dicot & Alien & A & $\mathrm{Cn}$ & - & - & + & - \\
\hline $\begin{array}{l}\text { Paspalum paspaloides } \\
\text { Scribner }\end{array}$ & Poaceae & Monocot & Alien & A & $\mathrm{Nt}$ & - & + & - & - \\
\hline Peganum harmala L. & Zygophyllaceae & Dicot & Native & $P$ & & + & - & - & + \\
\hline Phleum alpinum L. & Poaceae & Monocot & Native & $P$ & & - & + & + & + \\
\hline Phleum paniculatum Huds. & Poaceae & Monocot & Native & A & & + & + & - & - \\
\hline Phragmites australis Trin. & Poaceae & Monocot & Alien & $P$ & $\ln$ & - & + & - & - \\
\hline Plantago lanceolata $\mathrm{L}$. & Plantaginaceae & Dicot & Alien & $P$ & $\ln$ & + & + & + & + \\
\hline Plantago major $\mathrm{L}$. & Plantaginaceae & Dicot & Alien & $P$ & $\ln$ & + & + & + & + \\
\hline Poa angustifolia L. & Poaceae & Monocot & Alien & $P$ & $\mathrm{Nt}$ & + & + & + & + \\
\hline Poa annua L. & Poaceae & Monocot & Alien & A & $\ln$ & + & + & + & + \\
\hline Poa bulbosa L. & Poaceae & Monocot & Native & A & & + & + & + & + \\
\hline Poa pratensis $\mathrm{L}$. & Poaceae & Monocot & Alien & $P$ & $\mathrm{Nt}$ & - & + & - & - \\
\hline $\begin{array}{l}\text { Polygonum heterophyllum } \\
\text { Lindman }\end{array}$ & Polygonaceae & Dicot & Native & A & & + & + & + & + \\
\hline Polygonum hydropiper L. & Polygonaceae & Dicot & Alien & A & $\ln$ & - & - & + & + \\
\hline Polygonum plebejum R. Br. & Polygonaceae & Dicot & Native & A & & - & + & - & - \\
\hline $\begin{array}{l}\text { Polypogon fugax Nees } \\
\text { ex Steud. }\end{array}$ & Poaceae & Monocot & Alien & A & $\mathrm{Nt}$ & + & - & - & + \\
\hline Populus alba L. & Salicaceae & Dicot & Alien & $\mathrm{T}$ & $\mathrm{Nt}$ & - & - & - & + \\
\hline Portulaca oleracea L. & Portulacaceae & Dicot & Alien & A & $\mathrm{Nt}$ & - & + & - & + \\
\hline Potentilla nepalensis Hk. & Rosaceae & Dicot & Native & $P$ & & - & + & - & + \\
\hline Potentilla reptans $\mathrm{L}$. & Rosaceae & Dicot & Alien & $P$ & $\mathrm{Nt}$ & + & + & + & + \\
\hline Potentilla supina $\mathrm{L}$. & Rosaceae & Dicot & Alien & A & $\mathrm{Nt}$ & - & - & - & + \\
\hline Prunus armeniaca L. & Rosaceae & Dicot & Alien & $\mathrm{T}$ & Cs & - & - & - & + \\
\hline Prunus domestica L. & Rosaceae & Dicot & Alien & $\mathrm{T}$ & Cs & - & - & + & + \\
\hline Ranunculus arvensis $\mathrm{L}$. & Ranunculaceae & Dicot & Alien & A & In & - & - & + & + \\
\hline $\begin{array}{l}\text { Ranunculus laetus Wall. } \\
\text { ex Hk. f. and T. }\end{array}$ & Ranunculaceae & Dicot & Alien & $P$ & $\ln$ & - & - & + & - \\
\hline Ranunculus muricatus L. & Ranunculaceae & Dicot & Alien & A & $\ln$ & - & + & - & + \\
\hline Robinia pseudoacacia L. & Fabaceae & Dicot & Alien & $\mathrm{T}$ & $\ln$ & + & + & + & - \\
\hline Rorippa islandica Borbas & Brassicaceae & Dicot & Alien & $\mathrm{Aq}$ & $\mathrm{Nt}$ & + & - & + & + \\
\hline Rorippa sylvestris Besser. & Brassicaceae & Dicot & Native & $P$ & & + & + & + & + \\
\hline Rosa brunonii Lindl. & Rosaceae & Dicot & Alien & S & $\mathrm{Nt}$ & - & + & + & - \\
\hline Rubia cordifolia L. & Rubiaceae & Dicot & Alien & C & $\mathrm{Nt}$ & - & - & + & - \\
\hline $\begin{array}{l}\text { Rubus niveus Thunb. } \\
\text { non Wall. }\end{array}$ & Rosaceae & Dicot & Alien & S & $\mathrm{Nt}$ & - & + & + & - \\
\hline Rubus ulmifolius Schott. & Rosaceae & Dicot & Alien & S & $\ln$ & - & + & + & + \\
\hline Rumex dentatus $\mathrm{L}$. & Polygonaceae & Dicot & Alien & A & $\mathrm{Nt}$ & + & + & + & + \\
\hline Rumex nepalensis Spreng. & Polygonaceae & Dicot & Alien & $P$ & $\mathrm{Nt}$ & + & - & + & + \\
\hline $\begin{array}{l}\text { Salvia moorcroftiana Wall. } \\
\text { ex Bth. }\end{array}$ & Lamiaceae & Dicot & Native & $P$ & & + & - & + & + \\
\hline Sanguisorba minor Scop. & Rosaceae & Dicot & Alien & $P$ & $\mathrm{Nt}$ & - & - & + & - \\
\hline Scandix pecten-veneris L. & Apiaceae & Dicot & Alien & A & $\mathrm{Nt}$ & - & + & + & - \\
\hline Scirpus setaceus L. & Cyperaceae & Monocot & Native & $P$ & & - & + & - & - \\
\hline
\end{tabular}


Table 1 Conspectus of plant species in various habitats of Srinagar city (Continued)

\begin{tabular}{|c|c|c|c|c|c|c|c|c|c|}
\hline Sclerochloa dura P. Beauv. & Poaceae & Monocot & Native & $P$ & & + & - & - & - \\
\hline Scorozonera virgata DC. & Asteraceae & Dicot & Native & $P$ & & - & - & + & - \\
\hline Scrophularia lucida Hk. f. & Scrophulariaceae & Dicot & Native & $P$ & & - & - & + & - \\
\hline Setaria viridis P. Beauv. & Poaceae & Monocot & Alien & A & In & - & + & - & - \\
\hline Siegesbeckia orientalis L. & Asteraceae & Dicot & Alien & A & $\ln$ & - & - & + & - \\
\hline Sisymbrium loesellii L. & Brassicaceae & Dicot & Alien & A & $\ln$ & + & + & + & + \\
\hline Sisymbrium officinale Scop. & Brassicaceae & Dicot & Native & A & & - & - & + & + \\
\hline Solanum nigrum L. & Solanaceae & Dicot & Native & A & & + & - & + & + \\
\hline Sonchus asper Hill & Asteraceae & Dicot & Native & A & & - & - & + & - \\
\hline Sonchus oleraceus L. & Asteraceae & Dicot & Native & A & & - & + & + & + \\
\hline Sorghum halepense Pers. & Poaceae & Monocot & Alien & $P$ & $\ln$ & - & + & + & - \\
\hline Stachys floccosa Benth. & Lamiaceae & Dicot & Native & $P$ & & + & - & + & - \\
\hline Stellaria media Cyr. & Caryophyllaceae & Dicot & Alien & A & $\ln$ & + & + & + & + \\
\hline Taraxacum officinale Weber & Asteraceae & Dicot & Alien & $P$ & $\ln$ & + & + & + & + \\
\hline Torilis japonica DC. & Apiaceae & Dicot & Alien & A & $\mathrm{Nt}$ & + & + & + & + \\
\hline Torilis leptophylla Reichb. f. & Apiaceae & Dicot & Alien & A & $\mathrm{Nt}$ & + & + & - & + \\
\hline $\begin{array}{l}\text { Tragopogon kashmirianus } \\
\text { Gurcharan singh }\end{array}$ & Asteraceae & Dicot & Native & B & & + & - & + & + \\
\hline Trifolium pratense L. & Fabaceae & Dicot & Alien & $P$ & $\ln$ & + & + & + & + \\
\hline Trifolium repens $\mathrm{L}$. & Fabaceae & Dicot & Alien & $P$ & $\ln$ & + & + & + & + \\
\hline Trigonella foenum-graecum L. & Fabaceae & Dicot & Alien & A & Cs & - & - & - & + \\
\hline Tulipa clusiana DC. & Liliaceae & Monocot & Native & $P$ & & - & - & + & - \\
\hline Tulipa lanata E. Regel & Liliaceae & Monocot & Alien & $P$ & $\mathrm{Nt}$ & - & - & + & - \\
\hline Tussilago farfara $\mathrm{L}$. & Asteraceae & Dicot & Native & $P$ & & - & - & - & + \\
\hline Urtica dioica $\mathrm{L}$. & Urticaceae & Dicot & Alien & $P$ & $\ln$ & + & + & + & + \\
\hline Valerianella eriocarpa Desv. & Valerianaceae & Dicot & Native & A & & - & + & + & + \\
\hline Verbascum thapsus L. & Scrophulariaceae & Dicot & Alien & B & $\ln$ & + & + & + & - \\
\hline Verbena officinalis L. & Verbenaceae & Dicot & Native & A & & + & + & + & + \\
\hline Veronica agrestis $\mathrm{L}$. & Scrophulariaceae & Dicot & Native & A & & + & + & + & + \\
\hline Veronica anagallis-aquatica L. & Scrophulariaceae & Dicot & Alien & A & $\mathrm{Nt}$ & - & + & - & - \\
\hline Veronica persica Poir. & Scrophulariaceae & Dicot & Alien & A & $\ln$ & + & + & + & + \\
\hline Veronica stewartii Penn. & Scrophulariaceae & Dicot & Native & A & & - & + & + & - \\
\hline Vicia angustifolia L. & Fabaceae & Dicot & Native & A & & + & + & + & + \\
\hline Vicia sativa $\mathrm{L}$. & Fabaceae & Dicot & Alien & A & $\mathrm{Cn}$ & - & + & - & - \\
\hline Viola indica Bkr. & Violaceae & Dicot & Native & $P$ & & - & - & + & - \\
\hline Vulpia myuros Gmel. & Poaceae & Monocot & Alien & A & $\ln$ & + & + & + & + \\
\hline Xanthium spinosum $\mathrm{L}$. & Asteraceae & Dicot & Alien & A & $\ln$ & + & + & + & + \\
\hline Xanthium strumarium L. & Asteraceae & Dicot & Alien & A & $\ln$ & + & - & + & + \\
\hline
\end{tabular}

Growth form: A, annual herb; B, biennial herb; P, perennial herb; Ss, sub-shrub; S, shrub; T, tree; Aq, aquatics; C, climber; L, liana; Ps, parasitic herb. Invasion status: $\mathrm{Cs}$, casual aliens; $\mathrm{Cn}$, casual or naturalised aliens; $\mathrm{Nt}$, naturalised aliens; In, invasive aliens; (+) presence of a species; $(-)$ absence of a species.

\section{Flora of orchards}

The present study revealed the presence of 141 species belonging to 110 genera and 39 families (Table 1). Six families namely Asteraceae (29 species), Poaceae (14 species), Fabaceae (12 species), Brassicaceae (10 species), Lamiaceae and Rosaceae (8 species each) accounted for
$57.45 \%$ of the species (Table 2). Eighteen (46.15\%) families were represented by one species each, seven families were represented by two species each, five families by three species each, two families (Polygonaceae and Boraginaceae) were represented by four species and the other one family (Scrophulariaceae) by five species. Out of the 110 genera 
Table 2 Taxonomic structure of the floras of different habitat types in Srinagar city

\begin{tabular}{|c|c|c|c|c|c|}
\hline \multirow{2}{*}{ Family } & \multicolumn{5}{|c|}{ Number of species } \\
\hline & Clubbed & Graveyards & Grasslands & Orchards & Wastelands \\
\hline Asteraceae & 35 & 23 & 19 & 29 & 20 \\
\hline Poaceae & 27 & 10 & 22 & 14 & 16 \\
\hline Fabaceae & 16 & 6 & 10 & 12 & 8 \\
\hline Brassicaceae & 12 & 7 & 6 & 10 & 8 \\
\hline Rosaceae & 11 & - & 6 & 8 & 6 \\
\hline Scrophulariaceae & 9 & 3 & 6 & 5 & 4 \\
\hline Lamiaceae & 8 & 5 & 2 & 8 & 4 \\
\hline Apiaceae & 5 & 4 & 4 & 3 & 3 \\
\hline Boraginaceae & 5 & - & 2 & 4 & 3 \\
\hline Cyperaceae & 5 & - & 3 & 3 & - \\
\hline Polygonaceae & 5 & 3 & 3 & 4 & 4 \\
\hline Ranunculaceae & 5 & - & 2 & 2 & 3 \\
\hline Caryophyllaceae & 4 & 3 & 3 & 2 & - \\
\hline Malvaceae & 4 & 3 & - & 3 & - \\
\hline Chenopodiaceae & 3 & 2 & - & - & 2 \\
\hline Geraniaceae & 3 & 2 & 3 & 3 & 2 \\
\hline Iridaceae & 3 & 3 & - & - & - \\
\hline Liliaceae & 3 & - & - & 3 & - \\
\hline Amaranthaceae & 2 & 2 & - & - & 2 \\
\hline Convolvulaceae & 2 & - & - & - & 2 \\
\hline Equisetaceae & 2 & - & - & 2 & - \\
\hline Euphorbiaceae & 2 & - & - & 2 & - \\
\hline Onagraceae & 2 & - & 2 & 2 & - \\
\hline Plantaginaceae & 2 & 2 & 2 & 2 & 2 \\
\hline Rubiaceae & 2 & - & - & 2 & - \\
\hline Solanaceae & 2 & 2 & - & - & 2 \\
\hline
\end{tabular}

Only the families with two or more representatives are shown. (-) absence of a species.

only one genus, Medicago was represented by four species. Artemisia, Malva, Poa and Veronica were represented by three species each, 20 genera by two species each and $85(77.27 \%)$ genera were represented by just one species each.

Growth form and life span profile of orchard flora Out of 141 species, 130 (92.19\%) were herbaceous (60 annual, 11 biennial, 57 perennial, 1 aquatic and 1 climber), 2 were sub-shrubs and 4 were shrubs. Five tree species were also recorded in the study area (Figure 2).

Eighty-five (65.38\%) of the total 130 herbaceous species were alien and 45 (34.62\%) were native to the region. Out of two sub-shrubs, one was alien and other was native to the region. Out of four shrubs, three were alien and one was native. All the five tree species were alien to Kashmir.
Proportion of alien and native species Out of 141 species, 94 (66.67\%) were alien (Figure 3) and 47 (33.33\%) were native to Kashmir. Out of 94 alien species, 6 were casuals, 3 were casuals or naturalised; naturalised category included 35 species, and 50 (53.19\%) species represented invasive category (Table 3 ).

\section{Flora of wastelands}

Urban wasteland flora comprised 110 species, belonging to 89 genera and 36 families (Table 1). Five families, namely Asteraceae (20 species), Poaceae (16 species), Brassicaceae (8 species), Fabaceae (8 species) and Rosaceae (6 species) accounted for $52.73 \%$ of species (Table 2). Nineteen families were represented by single species each, six families by two species each, three families by four species each and three families were represented by three species each. The maximum number of species i.e. three species was of Poa and Potentilla. Seventeen 


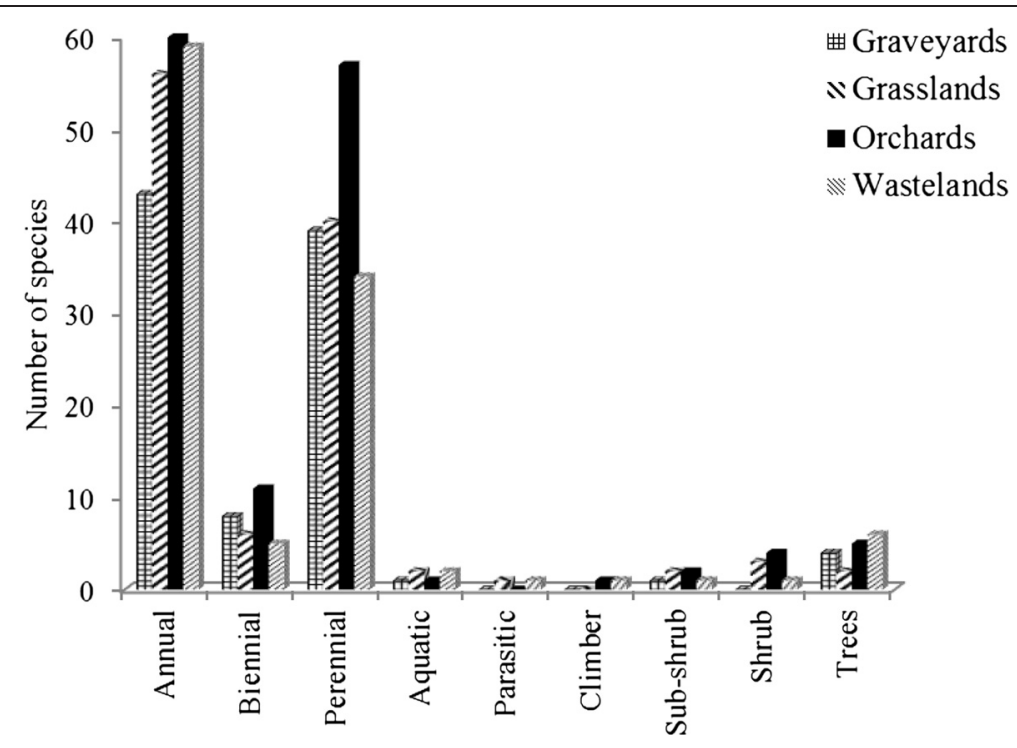

Figure 2 Habit and life form of the floras of various habitat types in Srinagar city.

genera were represented by two species each, and the remaining $70(78.65 \%)$ genera were represented by single species each.

Growth form and life span profile of wasteland flora Out of 110 species, 102 (92.59\%) were herbaceous (59 annual, 5 biennial, 34 perennial, 2 aquatic, 1 climber and 1 parasitic), besides 1 sub-shrub, 1 shrub and 6 tree species (Figure 2).

Proportion of alien and native species Out of 110 species, $74(67.27 \%)$ were alien (Figure 3) and 36 (32.73\%) were native to Kashmir. Casuals included 5 species; casuals or naturalised category included 2 species; 28 species were grouped under naturalised category and 39 species were grouped under invasive category (Table 3).

\section{Discussion}

This study revealed a preponderance of alien plant species across all habitat types. The proportion of alien species among habitats varied from $67.27 \%$ to $68.75 \%$, with a mean value of $67.86 \%$ and $64.84 \%$ in the clubbed species list. Several studies across the globe have reported a higher proportion of alien species in city floras. Pyšek (1998) in a study, involving 54 central European cities, reported a high proportion of alien plant species which ranged between $20 \%$ and $60 \%$. The proportion of alien species found in Ensenada, a fast-growing city located in the north-western peninsula of Baja California (Mexico) was also as high as 61\% (Garcillán et al. 2009). Kowarik (1990) also reported that Polish cities have an average of $50 \%$ to $70 \%$ alien plant species.

Across all habitats, four families such as Asteraceae, Poaceae, Fabaceae and Brassicaceae were having dominant contribution to the respective floras and they contributed

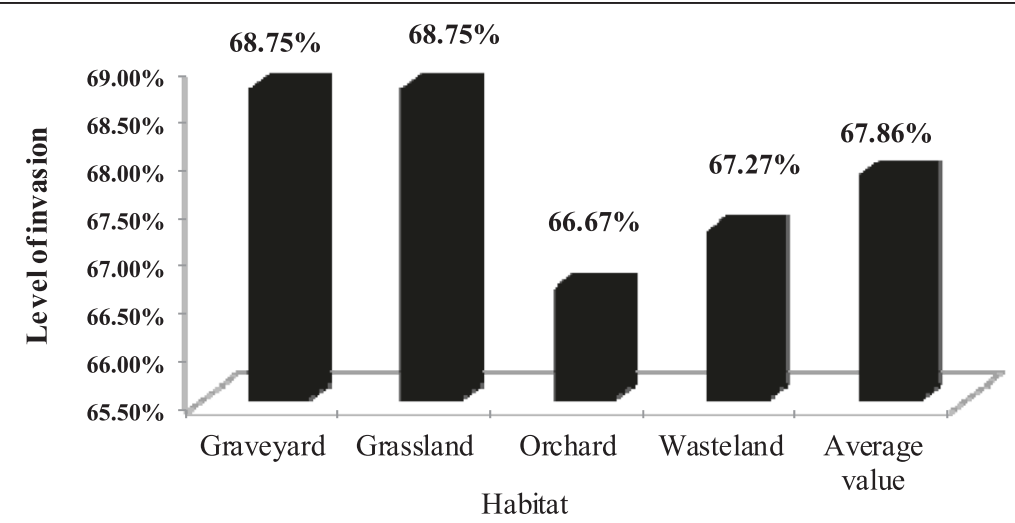

Figure 3 Comparison of level of alien plant invasion in various habitats of Srinagar city. 
Table 3 Comparison of alien plant species at different stages of invasion in various habitats of Srinagar city

\begin{tabular}{llllllll}
\hline Habitat & Aliens & \multicolumn{1}{l}{ Naturalised } & Invasive & $\begin{array}{l}\text { Total alien } \\
\text { species }\end{array}$ & Casual & $\begin{array}{l}\text { Casualised or } \\
\text { naturalised }\end{array}$ & $\begin{array}{l}\text { Total } \\
\text { species } \\
\text { habitat flora }\end{array}$ \\
\hline Graveyards & 4 & 1 & 24 & 37 & 66 & 68.75 & 96 \\
Grasslands & 2 & 2 & 33 & 40 & 77 & 68.75 & 112 \\
Orchards & 6 & 3 & 35 & 50 & 94 & 66.67 & 141 \\
Wastelands & 5 & 2 & 28 & 39 & 74 & 67.27 & 110 \\
\hline
\end{tabular}

$46.09 \%$ to $50.89 \%$ species to respective habitat floras. The clubbed species list was dominated by large global plant families which have a weedy tendency. The family that included the largest number of species was Asteraceae, followed by Poaceae, Fabaceae, Brassicaceae and Rosaceae. These five families accounted for $50.75 \%$ of alien plant species in the clubbed species list in the present study and have been reported to be dominant in other alien floras in Europe (e.g. Weber 1997; Pyšek et al. 2002; Celesti-Grapow et al. 2009) and in Asian countries (China Weber et al. 2008 and India Khuroo et al. 2012). By and large, all genera are represented equally and no genus or few of them dominated the species pool in urban landscapes.

This study revealed that plant species with herbaceous habitat dominated the clubbed species list as well as respective habitat floras and accounted for $92.46 \%$ of all species. The annual and perennial herbs were represented by $45.23 \%$ and $37.69 \%$ of plant species, respectively. This seems to commensurate with the reports that Kashmir Himalayan region has witnessed $25 \%$ introductions for landscaping and ornamenting purposes only (Khuroo et al. 2007). The dominance of herbaceous plant species, with annual and perennial lifespan, is in agreement with findings of Khuroo et al. (2007) who also reported preponderance of annual (32\% of all alien species) and perennial herbs (27\% of all alien species) in alien flora of Kashmir Himalaya. Wang et al. (2011) also reported the preponderance of herbaceous habit in naturalised plant species recorded in Beijing Municipality, China. The dominance of alien plant species with herbaceous habit found in urban landscapes of Kashmir valley is presumably due to high anthropogenic disturbance. In areas frequently disturbed, those species have an advantage that can colonise rapidly and make use of the additional resources created by the disturbance such as herbs or grasses with a low canopy height (Hobbs 1989; Lake and Leishman 2004).

\section{Conclusions}

Srinagar city is one of the fastest-growing urban centres where frequent disturbances due to rapid infrastructure development create bare niches and pave way for alien introductions. This is testified by the predominance of alien plant species in the flora of Srinagar city across habitats as found in the present study. The growing preference over time for using alien rather than native species in urban landscaping and ornamenting, despite the Kashmir Himalaya region being quite rich in native ornamentals, has exacerbated the urbanisation-mediated invasions. The role of disturbance, a hallmark of urbanisation, in facilitating plant invasions is also indicated by the dominance of fast-colonising herbaceous plant species as compared to other growth forms in almost all the investigated habitats. The rapid pace of urbanisation and consequent burgeoning invasion in the Srinagar city's studied habitats call for careful policy planning to devise effective strategies for the management of urban landscapes. In fact, using native plant species in developing the urban landscapes and new green zones should be the rule, not the exception.

\section{Abbreviation}

GRIN: Germplasm Resources Information Network.

\section{Competing interests}

The authors declare that they have no competing interest.

\section{Authors' contributions}

SMA and ZAR conceived and designed the study. SMA carried out the floristic sampling, and MAS and SQ helped with the coordination and contributed to discussions and data analysis. All authors read and approved the final manuscript.

\section{Acknowledgements}

SMA is thankful to university of Kashmir, Srinagar for the fellowship grant. Thanks are due to Dr. AR Naqshi, Ex-Reader Department of Botany, University of Kashmir, for his special help in plant identification. Thanks are due to the two anonymous reviewers for their critical appraisal of the manuscript.

\section{Author details}

${ }^{1}$ Department of Botany, University of Kashmir, Srinagar 190 006, Jammu and Kashmir, India. 'Department of Geography, Humboldt University of Berlin, Rudower Chaussee 16, 12489 Berlin, Germany. ${ }^{3}$ School of Architecture, Birmingham City University, The Parkside Building, 5 Cardigan Street, Birmingham B4 7BD, UK.

Received: 6 October 2014 Accepted: 29 December 2014

Published online: 31 January 2015

\section{References}

Bastin L, Thomas CD (1999) The distribution of plant species in urban vegetation fragments. Landscape Ecol 14:493-507

Bolger DT, Scott TA, Sauvajot RM, Potenza P, McCalvin C, Tran D, Mazzoni S, Soule ME (1997) Response of rodents to habitat fragmentation in coastal southern California. Ecol Appl 7:522-563 
Bolund P, Hunhammar S (1999) Ecosystem services in urban areas. Ecol Econ 29:293-301

Catling PM, Porebski ZS (1994) The history of invasion and current status of Glossy Buckthorn, Rhamnus frangula, in Southern Ontario. Can Field Nat 108:305-310

Celesti-Grapow L, Alessandrini A, Arrigoni PV, Banfi E, Bernardo L, Bovio M, Brundu G, Cagiotti MR, Camarda I, Carli E, Conti F, Fascetti S, Galasso G, Gubellini L, La Valva V, Lucchese F, Marchiori S, Mazzola P, Peccenini S, Poldini L, Pretto F, Prosser F, Siniscalco C, Villani MC, Viegi L, Wilhalm T, Blasi C (2009) The inventory of the non-native flora of Italy. Plant Biosys 143:386-430

Davis MA (2003) Biotic globalization: does competition from introduced species threaten biodiversity? Bioscience 53:481-489

Garcillán PP, Rebman JP, Casilla F (2009) Analysis of the non-native flora of Ensenada, a fast growing city in north-western Baja California. Urban Ecosyst 12:449-463

Germplasm Resources Information Network (GRIN) of the United States Department of Agriculture. http://www.ars-grin.gov/cgi-bin/hpgs/html/ taxgenform.pl. Accessed 20 Mar 2012

Guirado M, Pino J, Roda F (2006) Understory plant species richness and composition in metropolitan forest archipelagos: effects of forest size, adjacent land use and distance to the edge. Global Ecol Biogeogr 15:50-62

Haase D, Larondelle N, Andersson E, Artmann M, Borgstrom S, Breuste J, Gomez-Baggethun E, Gren A, Hamstead Z, Hansen R, Kabisch N, Kremer P, Langemeyer J, Rall EM, McPhearson T, Pauleit S, Qureshi S, Schwarz N, Voigt A, Wurster D, Elmqvist T (2014) A quantitative review of urban ecosystem service assessments: concepts, models, and implementation. AMBIO 43:413-433

Hobbs RJ (1989) The nature and effects of disturbance relative to invasions. In: Drake JA, Mooney HA, di Castri F, Groves RH, Kruger FJ, Rejmánek M, Williamson M (eds) Biological invasions: a global perspective. Wiley, Chichester, pp 389-405

Hobbs RJ, Huenneke LF (1992) Disturbance, diversity, and invasion: implications for conservation. Conserv Biol 6:324-337

Hobbs RJ, Yates CJ (2003) Impacts of ecosystem fragmentation on plant populations: generalising the idiosyncratic. Aust J Bot 51:471-488

Houlahan JE, Keddy PA, Makkay K, Findlay CS (2006) The effects of adjacent land use on wetland species richness and community composition. Wetlands 26:79-96

IUCN (International Union for the Conservation of Nature) (1999) IUCN guidelines for the prevention of biodiversity loss due to biological invasion. Species 31-32:28-42

Jauni M, Hyönen T (2010) Invasion level of alien plants in semi-natural agricultural habitats in boreal region. Agr Ecosyst Environ 138:109-115

Khuroo AA, Rashid I, Reshi Z, Dar GH, Wafai BA (2007) The alien flora of Kashmir Himalaya. Biol Invasions 9:269-292

Khuroo AA, Reshi ZA, Malik AH, Weber E, Rashid I, Dar GH (2012) Alien flora of India: taxonomic composition, invasion status and biogeographic affiliations. Biol Invasions 14:99-113

Kowarik I (1990) Some responses of flora and vegetation to urbanization in central Europe. In: Sukopp H, Hejny S, Kowarik I (eds) Urban ecology: plants and plant communities in urban environments, SPB, Academic Publ. bv, The Hague, The Netherlands pp 45-74

Kühn I, Klotz S (2006) Urbanization and homogenization-comparing the floras of urban and rural areas in Germany. Biol Conser 127:292-300

Kupfer JA, Malanson GP, Franklin SB (2006) Not seeing the ocean for the islands: the mediating influence of matrix-based processes on forest fragmentation effects. Global Ecol Biogeogr 15:8-20

Lake JC, Leishman MR (2004) Invasion success of exotic in natural ecosystems: the role of disturbance, plant attributes and freedom from herbivores. Biol Conserv 117:215-226

Levine MJ, Vilà M, D'Antonio MC, Dukes SJ, Grigulis K, Lavorel S (2003) Mechanisms underlying the impacts of exotic plant invasions. Proceedings of the Royal Society B: Biological Sciences 270:775-781

Miller JR (2005) Biodiversity conservation and the extinction of experience. Trends Ecol Evol 20:430-434

Miller JR (2006) Restoration, reconciliation, and reconnecting with nature. Biol Conserv 127:356-361

Miller JR, Hobbs RJ (2002) Conservation where people live and work. Conserv Bio 16:330-337

Pickett STA, Cadenasso ML, Grove JM, Nilon CH, Pouyat RV, Zipperer WC Costanza R (2001) Urban ecological systems: linking terrestrial ecological, physical, and socioeconomic components of metropolitan areas. Annu Rev Ecol Syst 32:127-157
Pyšek P (1998) Alien and native species in Central European urban floras: a quantitative comparison. J Biogeogr 25:155-163

Pyšek P, Hulme PE (2005) Spatio-temporal dynamics of plant invasions: linking pattern to process. Ecoscience 12:302-315

Pyšek P, Richardson DM, Rejmanek M, Webster GL, Williamson M, Kirschner J (2004) Alien plants in checklists and floras: towards better communication between taxonomists and ecologists. Taxon 53:131-143

Pyšek P, Sádlo J, Mandák B (2002) Catalogue of alien plants of the Czech Republic Preslia. Praha 74:97-186

Qureshi S (2010) The fast growing megacity Karachi as a frontier of environmental challenges: urbanization and contemporary urbanism issues. J Geogr Reg Plan 3:306-321

Qureshi S, Breuste JH, Jim CY (2013) Differential community and the perception of urban green spaces and their contents in the megacity of Karachi, Pakistan. Urban Ecosyst 16:853-870

Qureshi S, Haase D, Richard C (2014) The theorized urban gradient (TUG) method - a conceptual framework for socio-ecological sampling in complex urban agglomerations. Ecol Indic 36:100-110

Richardson DM, Pysek P, Rejmanek M, Barbour MG, Panetta FD, West CJ (2000) Naturalization and invasion of alien plants: concepts and definitions. Divers Distrib 6:93-107

Ricotta C, La Sorte FA, Pysek P, Rapson GL, Celesti-Grapow L, Thompson K (2009) Phyloecology of urban alien floras. J Ecol 97:1243-1251

Saunders DA, Hobbs RJ, Margules CR (1991) Biological consequences of ecosystem fragmentation: a review. Conserv Biol 5:18-32

Shah MA, Callaway RM, Shah T, Houseman GR, Pal RW, Xiao S, Luo W, Rosche C, Reshi ZA, Khasa DP, Chen S (2014) Conyza canadensis suppresses plant diversity in its nonnative ranges but not at home: a transcontinental comparison. New Phytol 202:1286-1296

Sullivan JJ, Timmins SM, Williams PA (2005) Movement of exotic plants into coastal native forests from gardens in northern New Zealand. New Zeal $\rfloor$ Ecol 29:1-10

Thompson K, McCarthy MA (2008) Traits of British alien and native urban plants. J Ecol 96:853-859

Turner WR, Nakamura T, Dinetti M (2004) Global urbanization and separation of humans from nature. BioScience 54:585-590

Wang H, Pujol JL, Meyerson LA, Qui JX, Wang XK, Ouyang ZH (2011) Biological invasions in rapidly urbanizing areas: a case study of Beijing, China. Biodivers Conserv 20:2483-2509

Weber E, Sun SG, Li B (2008) Invasive alien plants in China: diversity and ecological insights. Biol Invasions 10:1411-1429

Weber EF (1997) The alien flora of Europe: a taxonomic and biogeographic review. J Veg Sci 8:565-572

Wilson EO (1984) Biophilia. Harvard University Press, Cambridge

Wu SH, Hsieh CH, Chaw SM, Rejmanek M (2004) Plant invasions in Taiwan: insights from the flora of casual and naturalized alien species. Divers Distrib 10:349-362

\section{Submit your manuscript to a SpringerOpen ${ }^{\circ}$ journal and benefit from:}

- Convenient online submission

- Rigorous peer review

- Immediate publication on acceptance

- Open access: articles freely available online

High visibility within the field

- Retaining the copyright to your article

Submit your next manuscript at $\gg$ springeropen.com 